ESAIM: PROCEEDINGS, December 2013, Vol. 43, p. 108-116

S. Descombes, B. Dussoubs, S. Faure, L. Gouarin, V. Louvet, M. Massot, V. Miele, Editors

\title{
AN ASYNCHRONOUS WRITING METHOD FOR RESTART FILES IN THE GYSELA CODE IN PREVISION OF EXASCALE SYSTEMS*
}

\author{
O. Thomine ${ }^{1}$, J. Bigot ${ }^{2}$, V. Grandgirard ${ }^{1}$, G. Latu $^{1}$, C. Passeron $^{1}$ et F. \\ ROZAR $^{1,2}$
}

\begin{abstract}
Résumé. Ce travail concerne une procédure d'optimisation développée dans le code de calcul GYSELA (GYrokinetic SEmi-LAgrangian). L'optimisation de l'écriture de fichiers de reprise est nécessaire afin de palier aux crashs lors du calcul. Ces fichiers de redémarrage nécessitent un très grand espace mémoire, et ce d'autant plus que le maillage utilisé est gros. La bande passante entre les noeuds de calcul et le système de stockage étant limité, cela induit une non-scalabilité des écritures, ce qui limite actuellement la fréquence d'écriture de ces fichiers. Cette non-scalabilité se révèle être d'autant plus importante que les fichiers sont gros. En effet, ce temps d'écriture (une fois le réseau de communication saturé) dépend linéairement de la taille des fichiers à écrire. La nécessité d'introduire une méthode d'écriture désynchronisée se révèle donc d'une grande importance.

Un nouveau module a été développé dans GYSELA. Cette procédure asynchrone permet une écriture très fréquente de ces fichiers de redémarrage, en limitant un ralentissement important dû à la limitation de la bande passante. Cette méthode a été améliorée en introduisant une somme de contrôle de ces fichiers de redémarrage, afin de contrôler leur intégrité si leur utilisation s'avère nécessaire, et permettant un redémarrage automatique de la simulation après un crash.
\end{abstract}

\begin{abstract}
The present work deals with an optimization procedure developed in the full-f global GYrokinetic SEmi-LAgrangian code (GYSELA). Optimizing the writing of the restart files is necessary to reduce the computing impact of crashes. These files require a very large memory space, and particularly so for very large mesh sizes. The limited bandwidth of the data pipe between the computing nodes and the storage system induces a non-scalable part in the GYSELA code, which increases with the mesh size. Indeed the transfer time of RAM to data depends linearly on the files size. The necessity of non synchronized writing-in-file procedure is therefore crucial.

A new GYSELA module has been developed. This asynchronous procedure allows the frequent writing of the restart files, whilst preventing a severe slowing down due to the limited writing bandwidth. This method has been improved to generate a checksum control of the restart files, and automatically rerun the code in case of a crash for any cause.
\end{abstract}

\section{INTRODUCTION}

Modeling turbulent transport is a major goal in order to predict confinement issues in a tokamak plasma. The gyrokinetic framework considers a computational domain in five dimensions which allows for kinetic issues

\footnotetext{
* This work was partially supported by the G8-Exascale action NuFUSE contract. GYSELA computations were granted access to the HPC resources of Mésocentre d'Aix-Marseille Université, TGCC (Saclay/France) and IFERC (Rokkasho/Japan).

1 CEA Cadarache, FR-13108 Saint-Paul-les-Durance

2 Maison de la simulation, CEA Saclay, FR-91191 Gif sur Yvette
}

(C) EDP Sciences, SMAI 2013 
to be investigated in a plasma. Gyrokinetic simulations lead to huge computational needs. Up to now, the gyrokinetic code GYSELA has performed large simulations using from a few hundred up to tens of thousands of cores.

Our gyrokinetic model considers as main unknown a distribution function $\bar{f}$ that represents the density of ions at a given phase space position. This function depends on time and on 5 other dimensions. First, 3 dimensions in space $\mathbf{x}_{G}=(r, \theta, \varphi)$ with $r$ and $\theta$ the polar coordinates in the poloidal cross-section of the torus, while $\varphi$ refers to the toroidal angle. Second, velocity space has two dimensions: $v_{\|}$being the velocity along the magnetic field lines and $\mu$ the magnetic moment corresponding to the action variable associated with the gyrophase. Let us consider the gyro-center coordinate system $\left(\mathbf{x}_{G}, v_{\|}, \mu\right)$, then the non-linear time evolution of the $5 \mathrm{D}$ guiding-center distribution function $\bar{f}_{t}\left(r, \theta, \varphi, v_{\|}, \mu\right)$ is governed by the so-called gyrokinetic equation which reads $\mathrm{GBB}^{+} 06, \mathrm{Hah} 88$ in its conservative form:

$$
B_{\| s}^{*} \frac{\partial \bar{f}}{\partial t}+\nabla \cdot\left(B_{\| s}^{*} \frac{\mathrm{d} \mathbf{x}_{G}}{\mathrm{~d} t} \bar{f}\right)+\frac{\partial}{\partial v_{\|}}\left(B_{\| s}^{*} \frac{\mathrm{d} v_{\|}}{\mathrm{d} t} \bar{f}\right)=B_{\| s}^{*}\left(\mathcal{D}_{r}(\bar{f})+\mathcal{K}(\bar{f})+\mathcal{C}(\bar{f})+\mathcal{S}\right)
$$

where $\mathcal{D}_{r}(\bar{f})$ and $\mathcal{K}_{r}(\bar{f})$ are respectively a diffusion term and a Krook operator applied on a radial buffer region, $\mathcal{C}(\bar{f})$ corresponds to a collision operator (see $\mathrm{DPDG}^{+} 11$ for more details) and $\mathcal{S}$ refers to source terms (detailed in $\left.\mathrm{SGA}^{+} 10\right]$ ). The scalar $B_{\| s}^{*}$ corresponds to the volume element in guiding-center velocity space. The expressions of the gyro-center coordinates evolution $\mathrm{d} \mathbf{x}_{G} / \mathrm{d} t$ and $\mathrm{d} v_{\|} / \mathrm{d} t$ are not necessary. The useful information for this paper is that they depend on the $3 \mathrm{D}$ electrostatic potential $\Phi\left(\mathbf{x}_{G}\right)$ and its derivatives. In this Vlasov equation, $\mu$ acts as a parameter because it is an adiabatic motion invariant. Let us denote by $N_{\mu}$ the number of $\mu$ values. We have $N_{\mu}$ independent equations (Eq. 1) to solve at each time step. In the code, electrons are assumed adiabatic, i.e electron inertia is ignored. The electrostatic potential is determined by solving the self-consistent coupled quasi-neutrality equation

$$
-\frac{1}{n_{0}(r)} \nabla_{\perp} \cdot\left[\frac{n_{0}(r)}{B_{0}} \nabla_{\perp} \Phi(r, \theta, \varphi)\right]+\frac{1}{T_{e}(r)}\left[\Phi(r, \theta, \varphi)-\langle\Phi\rangle_{\mathrm{FS}}(r)\right] \mid=\tilde{\rho}(r, \theta, \varphi)
$$

The r.h.s of Eq. (2) is defined as $\tilde{\rho}=\frac{2 \pi}{n_{0}(r)} \int B(r, \theta) d \mu \int \mathrm{d} v_{\|} J_{0}\left(k_{\perp} \sqrt{2 \mu}\right)\left(\bar{f}-\bar{f}_{e q}\right)$ with $\bar{f}_{\text {eq }}$ representing a local ion Maxwellian equilibrium. The perpendicular operator $\nabla_{\perp}$ is defined as $\nabla_{\perp}=\left(\partial_{r}, \frac{1}{r} \partial_{\theta}\right)$. $B(r, \theta)$ represents the magnetic field with $B_{0}$ being its value at the magnetic axis. The radial profiles $n_{0}(r)$ and $T_{e}(r)$ correspond respectively to the equilibrium density and the electron temperature. $J_{0}$ which denotes the Bessel function of first order reproduces the gyro-average operation in Fourier space, $k_{\perp}$ being the transverse component of the wave vector. $\langle\cdot\rangle_{\mathrm{FS}}$ denotes the flux surface average defined as $\langle\cdot\rangle_{\mathrm{FS}}=\int \cdot \mathcal{J}_{\mathrm{x}} \mathrm{d} \theta \mathrm{d} \varphi / \int \mathcal{J}_{\mathrm{x}} \mathrm{d} \theta \mathrm{d} \varphi$ with $\mathcal{J}_{\mathrm{x}}$ the jacobian in space of the system.

Therefore, GYSELA code is a global nonlinear electrostatic code which solves the gyro-kinetic equation in a five dimension phase space with a semi-Lagrangian method $\mathrm{GBB}^{+} 06, \mathrm{GSG}^{+} 08$. The Semi-Lagrangian time integration technique SRBG99 couples the Lagrangian and Eulerian points of view. The main advantage offered by the semi-Lagrangian technique is that time steps are not restricted by the CFL condition. We combine this scheme with a second order in time Strang splitting method.

The code is written in Fortran 90 and parallelized by using an hybrid MPI/OpenMP paradigm. Large data structures are used in Vlasov and quasi-neutrality solver: the $5 \mathrm{D}$ data $\bar{f}$, and the electric potential $\Phi$ which is a $3 \mathrm{D}$ data structure along with some derivatives of the gyro-average of $\Phi$. Let $N_{r}, N_{\theta}, N_{\varphi}, N_{v_{\|}}, N_{\mu}$ be respectively the number of points in each dimension $r, \theta, \varphi, v_{\|}, \mu$, therefore the size of 5D and 3D data are $\left(N_{r} N_{\theta} N_{\varphi} N_{v_{||}} N_{\mu}\right)$ and $\left(N_{r} N_{\theta} N_{\varphi}\right)$.

With the continuous increase of the number of computers nodes, the crash probability of one or several nodes will increase in the future. The origins of these crashes have been studied during the last few years at the Los Alamos Laboratory reliability data sets (http://institutes.lanl.gov/data/fdata/). These crashes induce minor or major problems depending on the seriousness of the crash. Minor seriousness errors are frequently produced, 
approximatively every 8 hours on the 100 000-nodes Blue Gene computer $\mathrm{GCG}^{+} 07$. A severe crash on the other hand (inducing a code interruption), is produced every 7 to 10 days on the same computer $\mathrm{GCG}^{+} 07$. In Exascale prevision, the time between two successive crashes (hardware, software, network etc.) will be reduced from 3 to 26 minutes SG07.VS09. If the crash probability is too high, the numerical codes need to be resilient enough to recover the simulation after each crash. A compromise is needed between the time spent for restart file saving and the time spent in computing. If the restart files are saved too often, the time spent in restart file writing will impact the computation time, because of the interaction of the writing procedures and computing threads. Another major problem is the bandwidth between the storage array and the processing units. The size of the data to save increases with the mesh resolution. For large to very large data files, the bandwidth will decelerate the computation. Indeed, each node will wait for the end of the writing process before continuing the computation.

An asynchronous method for writing restart files has been implemented to tackle this problem. A checksum of the data array has been saved in the restart files to be able to detect if a crash happened during the saving and corrupted data. We will see that the asynchronous method induces computing and memory overheads. We will estimate this overhead and define the strategy to adopt in function of the file size to be written. A scientific computing dedicated study has been performed by Sancho SPJ04 which studies the feasibility of incremental check-pointing. Due to the data size needed for this method, we cannot use it for GYSELA. The incremental method needs a reference-field, which is in our case has a size of some TB. Another kind of check-pointing method consists of mirror checkpointing. This checkpointing algorithm consists in replicating the data contained on a node to a neighbor node. The major problem of this algorithm is the assumption that only one node can crash CD96. If two nodes (one containing the original data and the other containing the saved data) crash simultaneously, the failure cannot be recovered.

Several libraries have been developed to improve this writing feature. DejaVu RHV07] is one of the most well known asynchronous libraries. However, we do not use this library for the following two reasons which $D e j a V u$ does not allow. Alternating between two set of distinct datas allows us to be sure that at least one set of restart files is able to be used to restart the simulation. An other reason is to use the HDF5 library capabilities, like chunk decomposition of domain for very large fields $(>4 G B)$. A specific routine, who alternates writing between two distinct set of restart files, and with HDF5 file support, has been developed specifically for GYSELA. In the first section, we will present the problem which will appear in the next computer generation : which is an important increasing of crash probability during the runs. The optimum restart writing needed to minimize the computing time will be deduce in the second part. The profiling results obtained with this method will be shown in the third section. Finally, the conclusion and the future algorithm improvement will be present in the fourth section.

\section{Crash frequency on NeW architecture}

Increasing the number of nodes used for a parallel simulation induces an increase of the crash probability. The crash probability of one node is given by a Weibull law, with a mean lifetime for a node of $\lambda=4$ years, and an exponential value $k=0.78$ SG06]. This cumulated probability is:

$$
P(t)=1-\exp \left(-(t / \lambda)^{k}\right) .
$$

In the case of $N$ nodes, this probability reads:

$$
P_{N}(t)=1-\left(\exp \left(-(t / \lambda)^{k}\right)\right)^{N} .
$$

The mean computing time between 2 crashes with a success probability of $s \in[0,1[$ is given in equation (5) and is shown on figure 1 for a probability of success of $10 \%$.

$$
\bar{\tau}_{s}(N)=\lambda\left(\ln \left(\frac{1}{1-s}\right) \frac{1}{N}\right)^{1 / k} .
$$




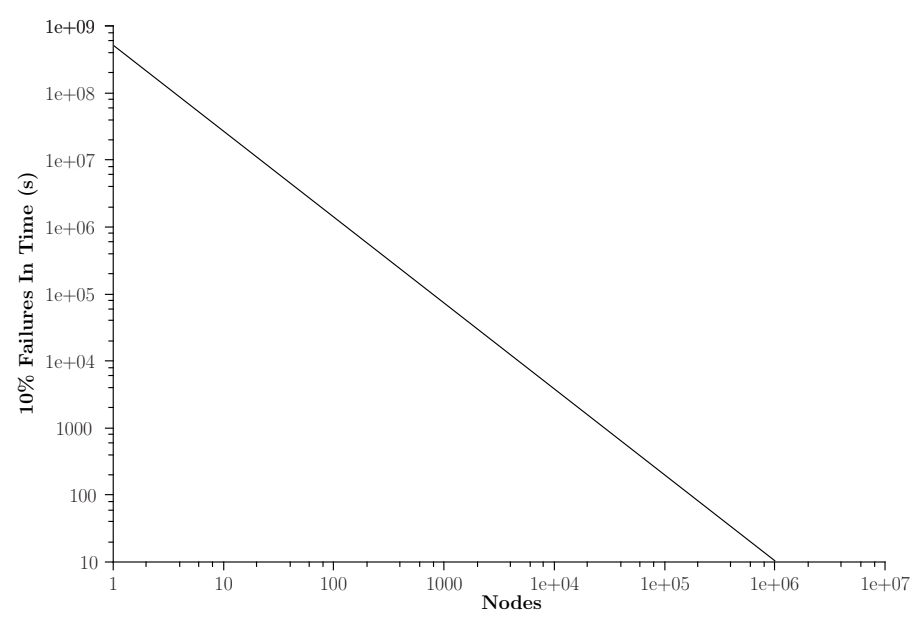

FiguRE 1. Evolution of mean computing time with a probability of success of $10 \%$ in function of the number of nodes.

For a large number of nodes $N=10^{5}$, this mean time $\bar{\tau}_{s}$ is of the order of the minute. For this reason, an upgrade of GYSELA that allows fault tolerance is very important.

Until now GYSELA used to write restart files only at the end of each run. If a crash appeared during the simulation, the whole run had to be re-computed. The solution has consisted in writing many restart files while GYSELA is running. With this procedure, when a crash occurs, the time that is lost is of the order of the time between two restart files savings. The checksum integrated in each restart file allows to make sure of their integrity. In this case, a new run with these restart files is launched, as shown on Fig. 2. To prevent issues during writing (file system failure for example), the initial restart file is not erased to preserve at least one non-corrupted file. In the case of a crash during restart file writing, the files will be corrupted. The next run cannot use these files as initialization data. So the last run has to be completely re-run, as shown on Fig. 3 .

\section{The ASYNCHRonous Method USED IN THE GYSELA CODE}

\subsection{Synchronous and asynchronous methods}

Until now, the restart files were written synchronously and used the HDF5 library due to its direct access capability to the data stored. The GYSELA code waits for the end of this writing procedure before it resumes computing. This waiting time is lost CPU time. For very large files to write, the delay due to the data transfers from local memory to file system is a big penalty.

The method used to desynchronize this writing procedure consists in launching a new thread dedicated to the data writing. During this file writing, the computing can continue. The major problem due to this method is the concurrent access to the memory containing the fields to write, between the computing subroutines and the writing thread activity. To prevent these concurrent accesses, a copy of the fields to be written is necessary. This copy is the overhead of this method: CPU time to copy the original fields to temporary fields, and memory needed to contain these fields. This overhead has been estimated and the results are shown in section 4. We show that the overhead of field copy before writing is of the order of $0.3 \%$ of the total simulation time. The major problem of this field copy is the memory needed for this operation. Most of the memory needed for each run is taken by the 5D field. We need two times more memory to keep a copy of this field. 


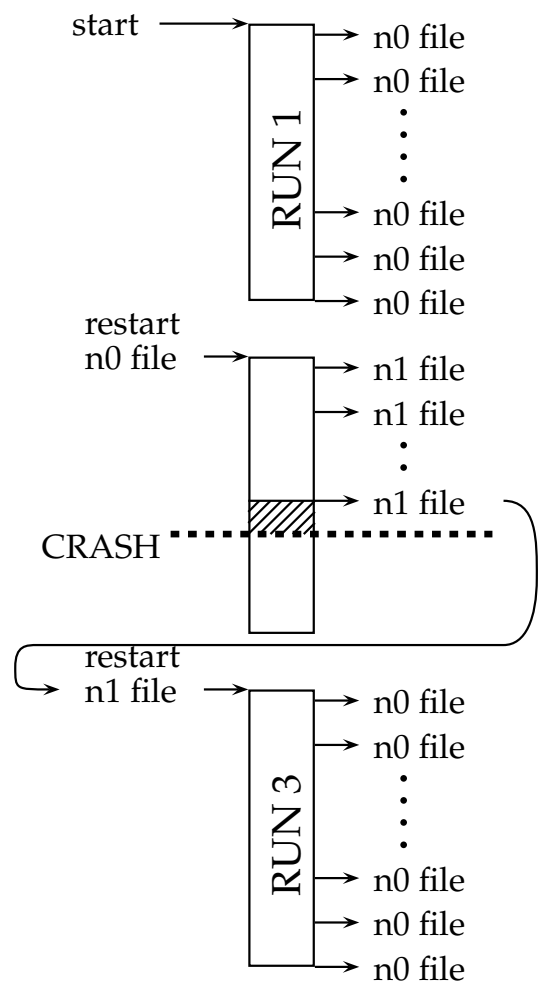

Figure 2. Crash during computation, restart files integrity checked.

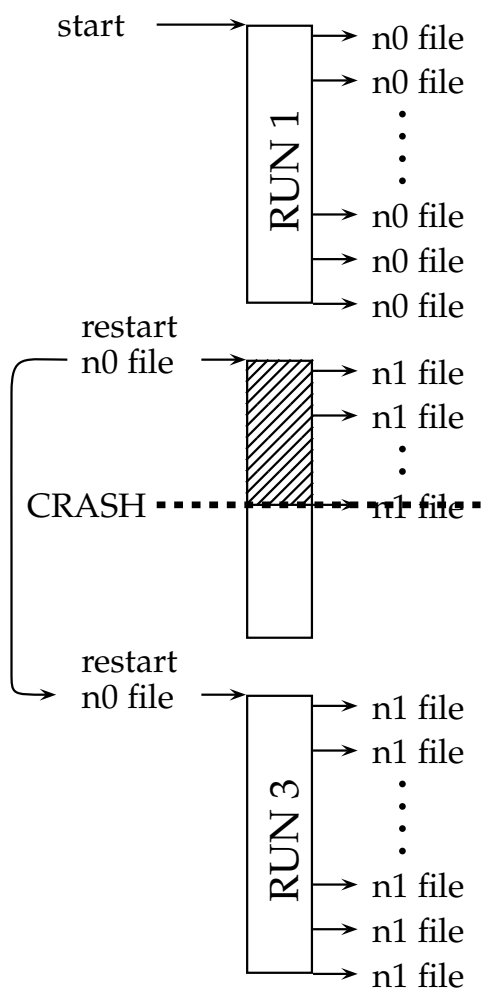

FiguRE 3. Crash during writing, restart files integrity unchecked.

\subsection{Optimizing the number of restart file savings}

One can deduce the optimal number of restart files during each simulation to minimize the average time which is lost due to asynchronous method. Let us suppose that the crash probability during an entire simulation is $\mathcal{P}$ (depending on the mean node lifetime, the number of nodes and the simulation time). Let $T_{e}$ be the time needed to write one restart file, $N_{e}$ is the parameter to optimize, namely the number of restart writing files in one run, and $\alpha$ a coefficient $(\in[0,1])$ which determines the CPU load needed for the writing procedure (in particular to compress and copy the fields to the temporary memory). We can consider that this parameter is equal to unity for synchronous writing. In one run, the time between two saving procedures is $\Delta T_{e}=T_{r} / N_{e}$, with the duration of one run being: $T_{r}=T_{c}+\alpha T_{e} N_{e}$. We can deduce the crash probability in a run, during the files writing with the asynchronous method as: $\mathcal{P}_{W}^{a s}=\mathcal{P} N_{e} T_{e} / T_{r}$, and out of the restart files writing as: $\mathcal{P}_{N W}^{a s}=\mathcal{P}\left(1-N_{e} T_{e} / T_{r}\right)$. The mean time lost in this case is $T_{r} / 2$ (Fig. 3). In the other case, i.e. when the crash does not occur during the restart file writing, the lost time is on average: $\Delta T_{e} / 2$ (Fig. 2). We can thus deduce the mean simulation time $\bar{\tau}_{s}$ in function of $N_{e}$ :

$$
\bar{\tau}=\underbrace{N_{t} T_{c}}_{\begin{array}{c}
\text { Simulation } \\
\text { CPU time }
\end{array}}+\underbrace{\mathcal{P}_{W}^{a s} \frac{T_{r}}{2}}_{\begin{array}{c}
\text { lost time } \\
\text { due to crash } \\
\text { during write }
\end{array}}+\underbrace{\mathcal{P}_{N W}^{a s} \frac{T_{r}}{N_{e}}}_{\begin{array}{c}
\text { lost time due } \\
\text { torash in-between } \\
\text { writings }
\end{array}}+\underbrace{N_{e} N_{t} \alpha T_{e}}_{\begin{array}{c}
\mathrm{CPU} \\
\text { overhead }
\end{array}},
$$




\begin{tabular}{|c|c|c|c|c|}
\hline Name & Mesh size & Number of nodes & Number of processors & Total file size \\
\hline SIM_1 & $2.1 \mathrm{G}$ & 16 & 256 & $18.0 \mathrm{~GB}$ \\
SIM_2 & $4.3 \mathrm{G}$ & 32 & 512 & $36.0 \mathrm{~GB}$ \\
SIM_3 & $8.5 \mathrm{G}$ & 64 & 1024 & $72.0 \mathrm{~GB}$ \\
SIM_4 & $17.0 \mathrm{G}$ & 128 & 2048 & $143.9 \mathrm{~GB}$ \\
SIM_5 & $36.1 \mathrm{G}$ & 256 & 4096 & $287.8 \mathrm{~GB}$ \\
SIM_6 & $68.2 \mathrm{G}$ & 512 & 8192 & $575.7 \mathrm{~GB}$ \\
SIM_7 & $136.4 \mathrm{G}$ & 1024 & 16384 & $1.1 \mathrm{~TB}$ \\
SIM_8 & $272.7 \mathrm{G}$ & 2048 & 32768 & $2.3 \mathrm{~TB}$ \\
\hline
\end{tabular}

TABLE 1. Simulations parameters used for GYSELA weak scaling.

with $N_{t}$ the number of runs during the simulation. We can easily show that the lowest value of $\bar{\tau}$ is obtained for the following value of $N_{e}$ :

$$
N_{e}=\sqrt{\frac{\mathcal{P} T_{c}}{T_{e}\left(2 \alpha N_{t}+\mathcal{P}\right)}} .
$$

Including the previous result to Eq. (6) gives the minimal simulation time $\bar{\tau}_{\text {MIN }}$ in function of $N_{t}, T_{c}, T_{e}, \mathcal{P}$ and $\alpha$. For a unique simulation, i.e. for $N_{t}, T_{c}, T_{e}, \mathcal{P}$ fixed, we can show that $\bar{\tau}_{\text {MIN }}$ increases when $\alpha$ increases. With $\alpha=1$, which corresponds to the synchronous method, $\bar{\tau}_{\text {MIN }}$ is maximum, as expected.

\section{Profiling Results}

To compare the saved time between synchronous and asynchronous methods, weak-scaling simulations have been performed on the HELIOS machine at CSC, Rokkasho, Japan. This Bullx B510 computer contains 70560 thin cores, which constitutes a 1.52 PFlops computing power. The bandwidth of data transfer towards the file system is about $20 \mathrm{~GB} / \mathrm{s}$. In our case (weak scaling), each node contains the same number of points. Using this scaling method allows us to increase the writing time, while keeping the same mesh point per node $\left(N_{R}=128, N_{\theta}=128, N_{\phi}=128, N_{v / /}=64, N_{\mu}=1\right.$, witch correspond to $134 M$ of points per node). The mesh size depending on the number of node is $N_{\mu}$. The uncompressed restart file size is $1.12 \mathrm{~GB}$. The simulation parameters are given in Table 1. Each MPI process is deployed on one node, the OpenMP parallelization allows each MPI process to use the 16 cores within the nodes. Three sets of simulations have been performed: one with the original synchronous method, one with the new asynchronous method, and one with no restart file writing. Each simulation contains 10 iterations, and saves the restart files every 4 iterations, meaning twice considered per simulation (for the synchronous and asynchronous cases, the last save is synchronous). The total data to copy from node memory to storage bay scales from 18 GB for 16 MPI processes to 2.3 TB for 2048 MPI processes. The computation time for each simulation is shown on Fig. 4 . We can see in Fig. 4 that multiplying by 128 the number of nodes induces a total time increase of the order of $55 \%$ if no restart file writing is asked, $55 \%$ if the asynchronous method is used and $115 \%$ with the synchronous method. These encouraging results show that the asynchronous method scales as well that without restart files writing. The relative efficiency of GYSELA is shown in Fig. 5

As we saw before, the asynchronous method induces an overhead due to the duplication of the data to write in addition to the thread dedicated to the writing. To estimate this overhead, the total time needed to copy the data has been estimated. These overheads are shown in Fig. 6. The time needed for the 5D-field copy is constant, which induce a perfect relative efficiency of this part. The time needed to launch the thread is constant too, and its impact is minor, compared to the copying procedure. For the above cases, the overhead required for the synchronous writing, the asynchronous writing and the no writing cases are similar. However, when we increase the size of the data to write (to approximatively $1 T B$ ), the bandwidth saturates. We can then see an important increase in the time needed to write the data. As the computing can continue in the asynchronous case while it cannot in the synchronous case, the total computing case strongly increases in the 


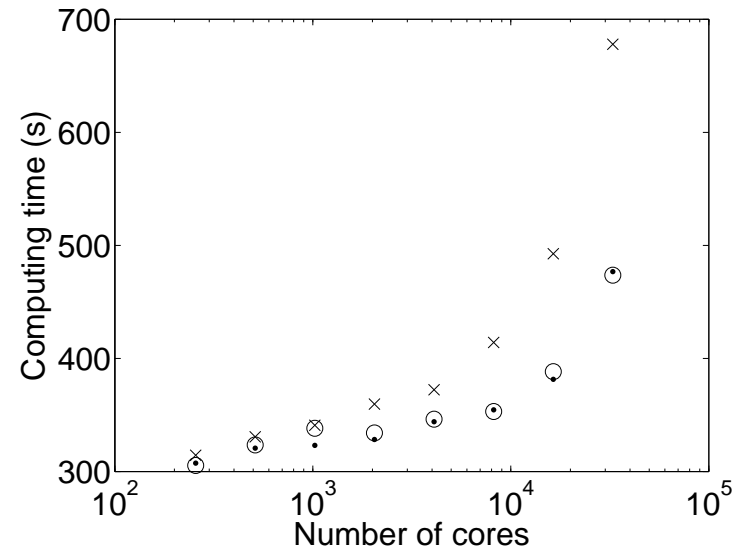

Figure 4. Total simulation time with synchronous (crosses), asynchronous (circles) and no writing (points) methods in function of number of cores.

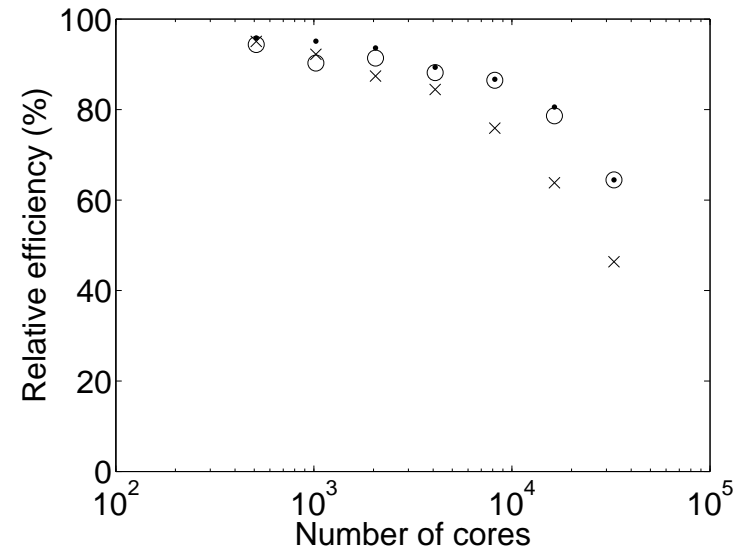

Figure 5. Relative efficiency obtained with synchronous (crosses), asynchronous (circles) and no writing (points) in function of number of cores.

synchronous case, but not in the asynchronous case, as shown in Fig. 4. However, as the bandwidth needed for the asynchronous writing is shared with the MPI communication, we can suppose that the bandwidth will not be optimal. We can see in Fig. 7 that we obtain a bandwidth of the same order of the asynchronous case in the synchronous case. This result denotes a low impact of file writing communication and MPI communications.

These comparisons have been made with a high frequency writing of the restart files. The optimum number of restart files to save during each simulation is detailed in section 3.2. In our case, this optimal number is of course near zero instead of 2 , due to very low probability of a crash on this low number of nodes.

The time used for both the synchronous and asynchronous writing procedures can be used to estimate the data transfer bandwidth. Table 2 summarizes the maximum time necessary to write one file (of about $175 \mathrm{MB}$ ). Using this time together with the total size of the restart files allows us to estimate the bandwidth. This estimation is of about $20 \mathrm{~GB} / \mathrm{s}$ for synchronous methods. In the asynchronous case, the MPI communications interact with the data writing and decreases the mean bandwidth. These bandwidths are in accordance with the nominal HELIOS specifications.

\section{Conclusion}

In this work, we have shown that the asynchronous method used for the restart file saving allows to circumvent the worst problems encountered in the synchronous cases. All the crashes that occurred have been resumed successfully and automatically due to this method. One can deduce that this asynchronous method is thus able to limit the lost time due to crashes without requiring severe constraints on the code structure and its complexity.

In this work we show that the asynchronous writing method used has a very low overhead, for files size from some GB to more than $1 T B$. This procedure has been adapted to easily restart the simulation in case of several crashes. We have also deduce the most efficiency frequency of restart file writing in function of crash probability of one node.

The optimum parameters presented in the second section will be relevant in the future generation of computers, who will have an high number of nodes $(>100000)$ and where the crashes will be more frequent than actually. Furthermore, the $\alpha$ parameter described in the second section is quasi-null in our case, because of 


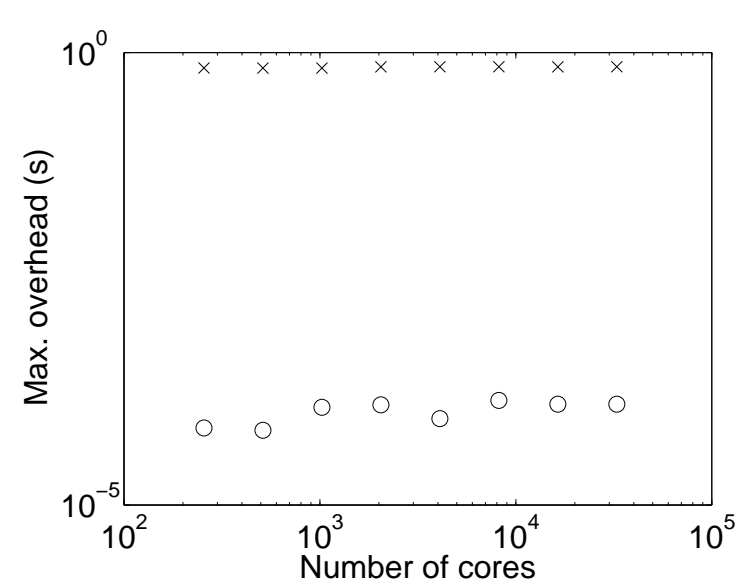

Figure 6. Overhead obtained with asynchronous method: 5D field copy (crosses) and thread launching (circles) in function of number of cores.

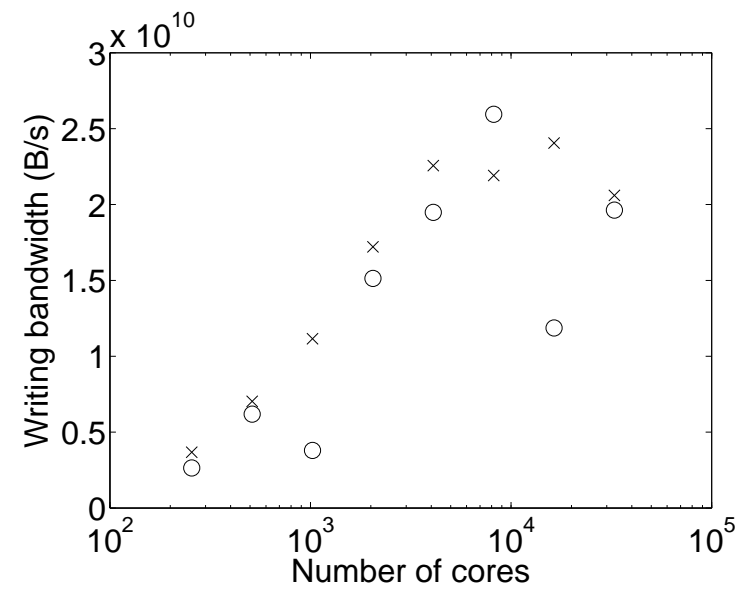

Figure 7. Bandwidth obtained with synchronous (crosses), asynchronous (circles) in function of number of cores.

\begin{tabular}{|c|c|c|c|c|}
\hline Writing method & Number of cores & Total file size & Max time (s) & Bandwidth \\
\hline synchronous & 256 & $18.0 G B$ & 5.23 & $3.7 \mathrm{~GB} / \mathrm{s}$ \\
synchronous & 512 & $36.0 G B$ & 5.48 & $7.0 \mathrm{~GB} / \mathrm{s}$ \\
synchronous & 1024 & $72.0 G B$ & 6.91 & $11.1 \mathrm{~GB} / \mathrm{s}$ \\
synchronous & 2048 & $143.9 G B$ & 8.96 & $17.2 \mathrm{~GB} / \mathrm{s}$ \\
synchronous & 4096 & $287.8 G B$ & 13.66 & $22.6 \mathrm{~GB} / \mathrm{s}$ \\
synchronous & 8192 & $575.7 G B$ & 28.14 & $21.9 \mathrm{~GB} / \mathrm{s}$ \\
synchronous & 16384 & $1.1 T B$ & 51.27 & $24.0 \mathrm{~GB} / \mathrm{s}$ \\
synchronous & 32768 & $2.3 T B$ & 119.73 & $20.6 \mathrm{~GB} / \mathrm{s}$ \\
\hline Max bandwidth & & & $24.0 \mathrm{~GB} / \mathrm{s}$ & \\
\hline \hline asynchronous & 256 & $18.0 G B$ & 7.30 & $2.6 \mathrm{~GB} / \mathrm{s}$ \\
asynchronous & 512 & $36.0 G B$ & 6.23 & $6.2 \mathrm{~GB} / \mathrm{s}$ \\
asynchronous & 1024 & $72.0 G B$ & 20.33 & $3.8 \mathrm{~GB} / \mathrm{s}$ \\
asynchronous & 2048 & $143.9 G B$ & 10.19 & $15.1 \mathrm{~GB} / \mathrm{s}$ \\
asynchronous & 4096 & $287.8 G B$ & 15.83 & $19.5 \mathrm{~GB} / \mathrm{s}$ \\
asynchronous & 8192 & $575.7 G B$ & 23.77 & $25.9 \mathrm{~GB} / \mathrm{s}$ \\
asynchronous & 16384 & $1.1 T B$ & 103.98 & $11.9 \mathrm{~GB} / \mathrm{s}$ \\
asynchronous & 32768 & $2.3 T B$ & 125.68 & $19.6 \mathrm{~GB} / \mathrm{s}$ \\
\hline Max bandwidth & & & $25.9 G B / \mathrm{s}$ & \\
\hline
\end{tabular}

TABLE 2. Estimated bandwidth.

uncompressed writing method used. In the case of compressed HDF5 method, this parameter will be higher than actually. 


\section{REFERENCES}

[CD96] T.-C. Chiueh and P. Deng. Evaluation of chekpoint mechanisms for massivly parallel machines. Proceedings of FTCS26, 1996.

[DPDG $\left.{ }^{+} 11\right]$ G. Dif-Pradalier, P. H. Diamond, V. Grandgirard, Y. Sarazin, J. Abiteboul, X. Garbet, Ph. Ghendrih, G. Latu, A. Strugarek, S. Ku, and C. S. Chang. Neoclassical physics in full distribution function gyrokinetics. 18(6):062309, 2011.

$\left[\mathrm{GBB}^{+}\right.$06] V. Grandgirard, M. Brunetti, P. Bertrand, N. Besse, X. Garbet, P. Ghendrih, G. Manfredi, Y. Sarazin, O. Sauter, E. Sonnendrucker, J. Vaclavik, and L. Villard. A drift-kinetic semi-lagrangian 4d code for ion turbulence simulation. Journal of Computational Physics, 2(217):395-423, 2006.

$\left[\mathrm{GCG}^{+}\right.$07] J.N. Glosli, K.J. Caspersen, J.A. Gunnels, D.F. Richards, Rudd R.E., and F.H. Streitz. Extending stability beyond cpu millenium: a micron-scale atomistic simulation of kelvin-helmoltz instability. In proceedings of the 2007 ACM/IEEE conference on supercomputing, 2007.

$\left[\mathrm{GSG}^{+} 08\right]$ V. Grandgirard, Y. Sarazin, X. Garbet, G. Dif-Pradalier, P Ghendrih, N. Crouseilles, G. Latu, E. Sonnendrucker, N. Besse, and P. Bertrand. Computing itg turbulence with a full-f semi-lagrangian code. Communications in Nonlinear Science and Numerical Simulation, 1(13):81 - 87, 2008.

[Hah88] T. S. Hahm. Nonlinear gyrokinetic equations for tokamak microturbulence. Physics of Fluids, 9(31):2670 - 2673, 1988.

[RHV07] J.F. Ruscio, M.A. Heffner, and S. Varadarajan. Dejavu: Transparent user-level checkpointing, migration, and recovery for distributed systems. IPDPS2007 Proceedings, 2007.

[SG06] B. Shroeder and G. Gibson. A large-scale study of failures in high-performance computing systems. Proceedings of ICDSN, 2006.

[SG07] B. Shroeder and G. Gibson. Understanding failure in petascale computers. Journal of Physics Conference Series: SciDAC, 2007.

$\left[\mathrm{SGA}^{+} 10\right]$ Y. Sarazin, V. Grandgirard, J. Abiteboul, S. Allfrey, X. Garbet, Ph. Ghendrih, G. Latu, A. Strugarek, and G. DifPradalier. Large scale dynamics in flux driven gyrokinetic turbulence. 50(5):054004, 2010.

[SPJ04] J.C. Sanlos, F. Petrini, and Fernandez J. On the feasibility of incremental checkpointing for scientific computing. Proceedings of IPDPS'04, 2004.

[SRBG99] E. Sonnendrucker, J. Roche, P. Bertrand, and A. Ghizzo. The semi-lagrangian method for the numerical resolution of the vlasov equation. Journal of Computational Physics, 2(149):201 - 220, 1999.

[VS09] E. Vivek Sarkar. Exascale software study: Software challenges in exascale systems. 2009. 